\title{
Machine Learning Approach and Waves Synchronization Improvement for the Localization of Atrial Flutter Circuit Based on the 12-leads ECG
}

\author{
O Meste $^{1}$, H K Azman ${ }^{1,2}$, D G Latcu ${ }^{3}$ \\ ${ }^{1}$ Université Côte d'Azur, CNRS, I3S, France \\ ${ }^{2}$ Universiti of Kuala Lumpur, Malaysia \\ ${ }^{3}$ Cardiology Department, Centre Hospitalier Princesse Grace, Monaco
}

\begin{abstract}
The localization of the Atrial flutter (AFL) is of great interest for ablation planification. Regardless the direction of rotation of the corresponding reentry loop, its left or right atrium origin needs to be known beforehand. This localization is usually performed by using visual inspection of the 12-leads standard ECG that could be computerized. The aim of the study is to automatically classify the corresponding averaged $F$-waves by using one to five simple features. The averaged $F$-wave is computed by introducing a new multi-lead extension of a SVD based method for the wave resynchronization.

A dataset of ECG recorded from 56 subjects and comprising 25 left AFL and 31 right AFL will train the classifier. From the averaged 12 leads $F$-wave, 3 groups (Gi) of features were extracted: G1-(min, max), G2-(integral of the negative, of the positive part), G3-(integral of the wave, integral of the absolute value of the wave). The logistic regression (LR) model is used for the supervised classifications.

The mean accuracy ranges for the three groups, without validations, are G1:[0.69-0.83], G2:[0.68-0.81], G3:[0.68-0.80] for one feature up to five. The maximum accuracy comes from G1 with five features and is equal to $93 \%$. The corresponding selected features are [ $\max (I)$, $\max (I I I), \max (V 3), \min (a V L), \min (V 5)]$.
\end{abstract}

\section{Introduction}

The localization of the source of Atrial Flutter (AFL) is of great importance for the guidance of the ablation procedure. Prior knowledge as to which atrium the circuit or focus is located will thus aid in the planning phase, effectively reducing operation time. It is well known that features visually extracted from the continuous ECG leads help practitioner for this task because they are shown to be linked to circuit localization [1], [2]. Although V1 is the most useful lead for distinguishing left from right atrial ori- gin [2], it doesn't reach the best performances that could be obtained by using extended combinations of leads.

It has been shown in [3] that the spatial variability of the F-waves could unveils from which chamber the AFL originates. The spatial variability is far from being addressed by a simple 12-leads ECG visual inspection used by practitioners. One goal of this paper is to refer to the regular practice to distinguish left from right atrial origin of the AFL. This practice mostly makes use of extremum values and polarities of the F-waves, considering simple combinations of multiple leads. In order to reject any source of disturbances that could impede a correct classification, a synchronous averaging is applied to the segmented F-waves automatically detected by using previous work [4]. The synchronous averaging is a very well known and efficient processing to remove noise but needs aligned waveforms.

This alignment, also addressed as a time delay estimation problem, has been considered in [5] as a problem of eigenvalues maximization in a context of one-dimensional multiple observations. In the context of 12-leads ECG system, the multiple delayed F-waves should be considered as a 12-dimensional alignment. Then, results from [5] is easily extended in this work to the $\mathrm{N}$-dimensional space in order to improve the accuracy of the delays estimation. The subsequent synchronous averaging provides reduced noise F-waves where features captured from visual inspection are in that case computerized. For each averaged Fwave and for each lead these computed features are : min, max, integral of the negative/positive parts, global integral, global integral of the absolute value.

Finally, according to the labeled right (R) or left (L) AFL cases evaluated during an ablation procedure, a machine learning algorithm is applied for a supervised classification with cross-validation performance assessment. Then, it is shown that with a limited number of features the prediction of the AFL localization can be performed. 


\section{Methods}

\subsection{F-waves alignment}

For the one-dimensional case :

$x_{i}(n)=s\left(n-\theta_{i}\right)+v_{i}(n), n=0, \ldots, N-1 ; i=1, \ldots, M$

where $s(n)$ is an unknown, deterministic signal, with energy $E_{s}$ and energy of its first derivative $E_{s^{\prime}}, \theta_{i}$ is random, zero mean, integer valued time delay with variance $\sigma_{\theta}^{2}$, and $v_{i}(n)$ is a zero-mean, white noise with variance $\sigma_{v}^{2}$, uncorrelated with $\theta_{i}$. It is shown in [5] that the estimation of the $\theta_{i}$ 's can be carried out by maximizing an eigenvalue-based criteria. The eigenvalues $\lambda_{i}$ are then computed from the intra-signal correlation matrix. From this work, an easily interpretable similar criteria is used and defined by :

$\Delta(\boldsymbol{\theta})=\frac{\lambda_{1}(\boldsymbol{\theta})}{\sum_{i=1}^{M} \lambda_{i}(\boldsymbol{\theta})}=\frac{E_{s}-\sigma_{\theta}^{2} E_{s^{\prime}}+\sigma_{v}^{2}}{E_{s}+N \sigma_{v}^{2}}=F(\boldsymbol{\theta})+B(\boldsymbol{\theta})$

Then $\Delta(\boldsymbol{\theta})$ is maximized when $\sigma_{\theta}^{2}$ is minimized. This minimization is indirectly performed by an iterative search of the best individual $\theta_{i}$ that maximizes (2). This expression can also be interpreted as the sum of a true function $F(\boldsymbol{\theta})$ added to zero-mean uncertainty $B(\boldsymbol{\theta})$ coming from approximations and other departures from model (1). This uncertainty impede the correct estimation of $\boldsymbol{\theta}$.

It can be assumed that in 12-leads ECG, each individual delay $\theta_{i}$ is equal across the different leads, even though $s(n)$ could be different. Then, these multiple observations of each $\theta_{i}$ should lead to a more accurate estimation. In order to benefit from for this property, it is proposed the following criteria :

$$
\nabla(\boldsymbol{\theta})=\frac{1}{12} \sum_{j=1}^{12} \Delta_{j}(\boldsymbol{\theta})=\frac{1}{12} \sum_{j=1}^{12} F_{j}(\boldsymbol{\theta})+\frac{1}{12} \sum_{j=1}^{12} B_{j}(\boldsymbol{\theta})
$$

Where $\Delta_{j}(\boldsymbol{\theta})$ stands for criteria (2) applied to the $j^{\text {th }}$ lead. Since each $\Delta_{j}(\boldsymbol{\theta})$ are bounded by $(0,1)$, so will be the $\nabla(\boldsymbol{\theta})$ values. The rational of this proposition is that since all the $F_{j}$ are maximum for the true $\boldsymbol{\theta}$ and the averaging of the uncorrelated $B_{j}(\boldsymbol{\theta})$ should tends toward zero as the number of leads increases, the estimation of $(\boldsymbol{\theta})$ will be more accurate.

It is easy to check that the more one lead is noisy, the less it will contribute to the optimization. Furthermore, because $E_{s^{\prime}}$ stands for the energy of the signal first derivative, the lead with the sharpest waveform will contribute more to the estimation. Finally, the shape changes that could be observed on given lead, caused by baseline contribution for instance, may also explain a lower contribution of the corresponding lead because of the spread of the eigenvalues spectrum and then a lower $\lambda_{1}$ value.
The performances of this approach can be depicted in Fig. 1 where the $\Delta$ values computed as in (2) are plotted. The corresponding aligned F-waves are displayed in Fig. 2 through their respective synchronous averages. From Fig. 1 it is clear that the estimation of the delays by maximizing $\nabla$ in (3) increases each individual $\Delta$. However, these $\Delta$ values are lower than the ones computed considering the leads as independent, providing different delays across the different leads. This latter result could appear better but is inconsistent with the underlying physical model.

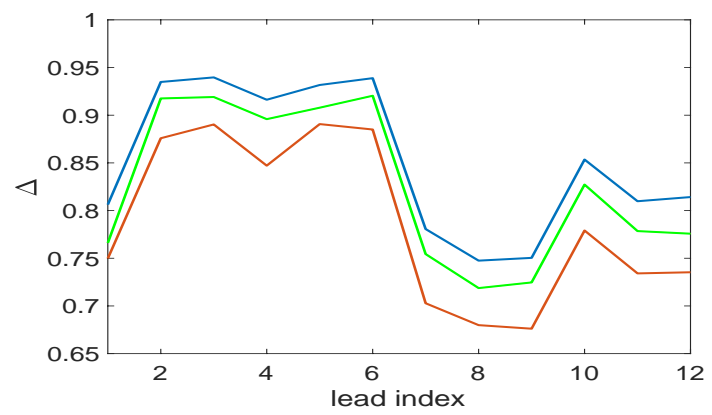

Figure 1. $\Delta$ values computed as in (2) over each lead, before (red), after (green) the proposed alignment and for the 12-leads plotted in Fig. 2. The blue trace corresponds to the $\Delta$ values optimized considering the leads independently.

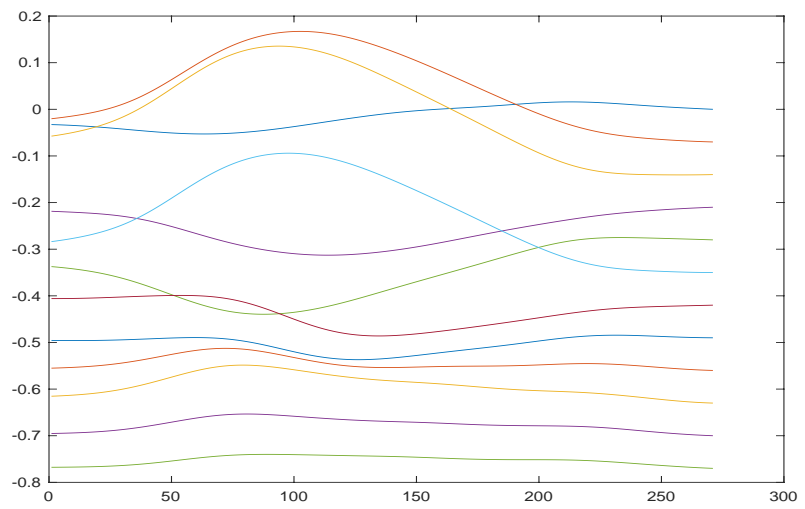

Figure 2. 12-leads averaged F-waves of a patient with left AFL. Traces from top to bottom correspond to I, II, III, aVR, aVL, aVF, V1, V2, V3, V4, V5, V6

\subsection{Supervised classification and features selection}

Many features could be extracted from the averaged Fwaves, taking into account all the ECG leads even though they are correlated. The visual inspection performed by practitioners shows the efficiency of the knowledge involved in their AFL classification. The visual inspection combines several salient characteristics such as the negative or positive peak values and also the isoelectric level for 
macro-reentry assessment. It is however difficult to visually combine these information, more or less exploited independently when the number of considered leads is large. The logistic regression classifier is a good candidate because it linearly combines a set of features, assuming that the labeling (right or left AFL) is a priori known. Non linear classifier are rejected in order to limit the overfitting due to the reduced number of observations.

Once the alignment process is achieved the averaged synchronous 12 leads F-waves are computed (see Fig. 2 and Fig. 3 for respective left and right AFL examples). For each lead and subject 3 groups (Gi) of features were extracted: G1-(min, max), G2-(integral of the negative, of the positive part), G3-(integral of the wave, integral of the absolute value of the wave). For each group Gi, 24 features are then computed to feed the learning algorithm. Combinations of features from different groups is not discussed in this study. A wrapper approach using an exhaustive search for feature selection is applied to maximize the mean classification accuracy computed over one to five features for each group $(\mathrm{Gi})$ applied to the 12 leads. The maximum number of features is also considered in the study. In order to assess the generalization ability of the best features combination a leave-one-out cross-validation (LOOCV) is also performed for comparison.

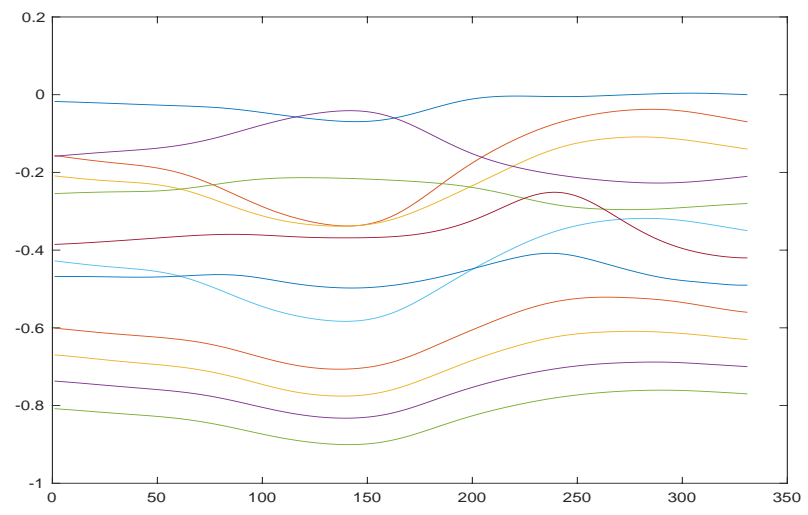

Figure 3. 12-leads averaged ECG of a patient with right AFL. Traces from top to bottom correspond to I, II, III, aVR, aVL, aVF, V1, V2, V3, V4, V5, V6

\section{Material}

A dataset of ECGs recorded from 56 subjects and comprising 25 left AFL and 31 right AFL will train the classifier. ECGs are acquired during catheter ablation operations at Centre Hospitalier Princesse Grace, Monaco. All signals were recorded at $f_{s}=2000 \mathrm{~Hz}$. A finite-impulse response notch filter at $50 \mathrm{~Hz}$ was applied to these signals to remove powerline interference, and were then filtered at $[0.5 ; 70] \mathrm{Hz}$ using type II Chebyshev high- and lowpass filters to remove baseline wander and high-frequency noises. Records with missing leads, low F-wave amplitudes, low atrioventricular block ratio $(<2$ : 1$)$ were excluded from the study. F-waves from each recording were detected and segmented, using a technique described in [4]. Only F-waves not overlapped within $\mathrm{T}$ waves were considered. The averaged number of segmented F-waves per subject is 64 with an average length of 360 samples. These segmented 12-leads F-waves are finally accurately aligned using the proposed method. It should be noticed that since the technique in [4] performs very well and the profiles of the F-waves are smooth, the impact of the alignment method proposed in this paper over the features is very weak.

\section{4. $\quad$ Results}

It is given in Fig. 4 the performances in term of accuracy of the classifier and for a variable number of features. It can be noticed that regardless the number of features the group G1 (min,max) exhibits the best classification and that the larger the number of features is the higher is the performance. However, the highest increase of performance is from one feature to two features. The best G1 combinations and the respective classification accuracies (\%) without or with LOOCV, for an increasing number of features is: $[\min (\mathrm{III})](84,80),[\max (\mathrm{I}), \min (\mathrm{V} 5)](89,85)$, $[\max (\mathrm{I}), \min (\mathrm{V} 3), \min (\mathrm{V} 5)](91,85),[\max (\mathrm{I}), \max (\mathrm{aVL})$, $\min (\mathrm{V} 4), \min (\mathrm{V} 5)](93,82),[\max (\mathrm{I}), \max (\mathrm{III}), \max (\mathrm{V} 3)$, $\min (\mathrm{aVL}), \min (\mathrm{V} 5)](93,86)$ Also, for the full set of 24 features, the accuracy without validation is $93 \%$ and $67 \%$ for the LOOCV. It can be observed that $\max (\mathrm{I})$ and $\min (\mathrm{V} 5)$ play a key role in the classification since they appear in almost all the best combinations. In addition, the result of the supervised classification is depicted in Fig. 5 where the result of the linear combination performed by the logistic regression is given. It can be observed that the two populations are well separated by the optimal threshold.

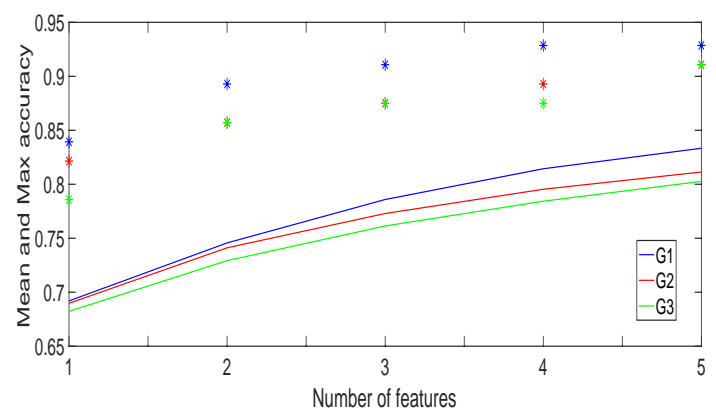

Figure 4. Mean and Max (*) accuracy for the Gi's 


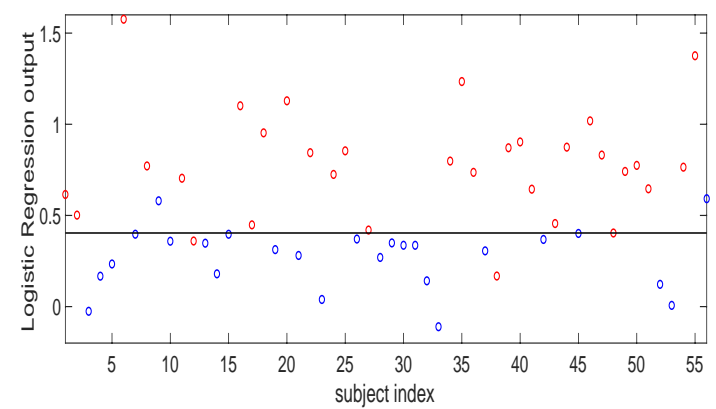

Figure 5. Output of the logistic regression for the best five features combination. The 56 subjects are depicted in red (right AFL) and blue (left AFL) colored circles. The black line stands for the optimal threshold separating the two classes.

\section{Discussion and conclusion}

A new multi-leads method that extends a single lead alignement technique has been proposed with a high potential for applications in the field of ECG analysis. It has been applied to F-waves recorded from right and left AFL subjects. It has been shown that based on a simple features extraction the classification of right and left AFL can be performed with high accuracy. The increase of the features number above two does not provide a real improvement with a risk of lack of generalization due to overfitting. These results need to be confronted to the expertise of practitioners even though the selected features are similar to those involved in visual inspection.

\section{References}

[1] Barbato G, Carinci V, Tomasi C, Frassineti V, Margheri M, Pasquale GD. Is electrocardiography a reliable tool for iden- tifying patients with isthmus-dependent atrial flutter ? Europace 2009;1071-1076.

[2] Medi C, Kalman JM. Prediction of the atrial flutter circuit location from the surface electrocardiogram. Europace $2008 \mathrm{Jul}$;10(7):78696.

[3] Kamarul Azman MH, Meste O, Kadir K, Latcu DG. Localizing atrial flutter circuit using variability in the vectorcardiographic loop parameters. In Computing in Cardiology, vol. 45. Sept. 2018.

[4] Kamarul Azman MH, Meste O, Kadir K. Detecting flutter waves in the electrocardiogram using generalized likelihood ratio test. In Computing in Cardiology, vol. 45. Sept. 2018.

[5] Laguna P, Garde A, Giraldo B, Meste O, Jané R, et al.. Eigenvaluebased time delay estimation of repetitive biomedical signals. Digital Signal Processing, Elsevier, 2018; (75) 107-119

Address for correspondence:

Pr. Olivier MESTE

Laboratoire I3S - CNRS - UNS

2000 route des Lucioles

06903 Sophia Antipolis cedex, FRANCE

E-mail address: olivier.meste@univ-cotedazur.fr 